\title{
The Application of Lab Environment as the External Disturbance in the 3-Stage IR Method for the Facile Discrimination of Danggui Formula Granules
}

\author{
Bi Xiaodong ${ }^{1,3,4}$, Ma Jingmei ${ }^{2,3,4}$, Tian Wei ${ }^{2,3,4}$, Peng Xinhua ${ }^{5}$, Gao Han ${ }^{5}$, Li Junshan ${ }^{5}$, \\ Niu Liying $1,3,4$, * \\ ${ }^{1}$ Department of Pharmacy, Hebei University of Chinese Medicine, Shijiazhuang, China \\ ${ }^{2}$ Department of Laboratory Center, Hebei University of Chinese Medicine, Shijiazhuang, China \\ ${ }^{3}$ Hebei Traditional Chinese Medicine Formula Granule Engineering \& Technology Research Center, Shijiazhuang, China \\ ${ }^{4}$ Traditional Chinese Medicine Formula Granule Research Center of Hebei Province University, Shijiazhuang, China \\ ${ }^{5}$ Shineway Pharmaceutical Group Ltd, Shijiazhuang, China
}

Email address:

niuliyingyy@163.com (Niu Liying)

${ }^{*}$ Corresponding author

\section{To cite this article:}

Bi Xiaodong, Ma Jingmei, Tian Wei, Peng Xinhua, Gao han, Li Junshan, Niu Liying. The Application of Lab Environment as the External Disturbance in the 3-Stage IR Method for the Facile Discrimination of Danggui Formula Granules. Modern Chemistry.

Vol. 6, No. 2, 2018, pp. 15-19. doi: 10.11648/j.mc.20180602.11

Received: June 13, 2018; Accepted: June 28, 2018; Published: July 23, 2018

\begin{abstract}
Formula granule (FG), as an improved preparation form of traditional liquid dedoction, is of increasing interest and hence it is necessary to develop facile analytical or discrimination methods. Based on the established 3-Stage IR method, a new external disturbance of lab environment (LE) in which room temperature (RT) is $25-30^{\circ} \mathrm{C}$ and humidity is $25-30 \%$, is introduced to test its applicability in discrimination of Danggui formula granules from different companies in which the LE is used to affect the prepared $\mathrm{KBr}$ pellet to produce enough variations for the 3S-IR method. Firstly, the 2D-spectra and pattern recognition algorithm of principal component analysis (PCA) and hierarchical cluster analysis (HCA) are used to select the analytical basis for 2D-COS IR. Secondly, LE-based 2D-COS IR is used for the discrimination. The newly-established LE-based IR can avoid the complex parts of programmed temperature and sampling in IR instrument, and achieve the discrimination of different Danggui Formula Granules.
\end{abstract}

Keywords: Formula Granule, Danggui, IR, Lab Environment, Discrimination

\section{Introduction}

Angelicae Sinensis Radix (called Danggui in Chinese, abbreviated as DG hereinafter) is officially recorded as traditional Chinese medicine (TCM) to possess the function of enriching blood, activating blood circulation, regulating menstruation and amenorrhoea, relieving pain and relaxing bowels [1, 2]. Due to its pharmacological effects [1, 3, 4], DG has been one of the commonly used ingradients in compound prescriptions, and also has been made into several dosage forms such as pills, dedoctions and oral liquids [1, 5-7].

Formula granule (FG) is made by spray drying of extracts of the effective compositions of the decoction of a single herbal medicine [8, 9]. Instead of boiling the mixture of herbal prescriptions for hours, the formula granule can be instantly dissolved in water by just adding one or several kinds of TCM according to the prescription, and hence provides much flexibility and convenience [9]. As the recently-emerging improved preparation form of traditional liquid dedoction, the corresponding standards for quality control of FGs is on the way [10]. Because of the involvement of modern extraction and preparation technology, especially the change of physical appearance of the TCM, it is of vital importance to guarantee the validity of manufacture and also identify the sources in quality control of FGs by means of modern analytical methods. 
Componential analysis, used in the analysis of FGs, usually includes two kinds: (1) liquid chromatography (LC)-based methods [11-14] for providing much information of detailed compositions, namely micro-fingerprint. LC-based method is able to be quatitative but meanwhile time-consuming, (2) IR-based methods [8, 11, 15-19] for providing holistic features, namely macro-fingerprint. IR-based methods are rapid but mostly qualitative due to the overlapping spectra of complex compositions. By virtue of the development of spectrometry technology including derivative IR spectrometry (DE-IR) and the two dimensional correlation IR spectrometry (2D-COS IR), and with the assistance of data processing methods such as pricinple component analysis (PCA), IR-based methods have seen tremendous applications in the differentiation and identification of TCM [15]. Among the established IR-based methods, the most noteworthy one is the three-stage infrared spectroscopy identification method (3S-IR) developed by Sun [8, 15-20]. The 2nd derivative IR (2D-IR) spectroscopy is used for high-resolved and in-depth analysis of the FT-IR. The 2D-COS IR spectroscopy introduces external interferences to help providing more detailed information when the system gives responses. However, the external disturbance usually used in 3S-IR is continuous changing of temperature, and this depends on the temperature control unit in the FT-IR instrument, which is not very common and somewhat expensive in common labs. Since IR-based methods can provide both holistic feature and detailed information at the same time in rapid differentiation of TCM and TCM preparations, it is necessary to explore new ways to implement the external disturbance for wide and convenient application of 3S-IR.

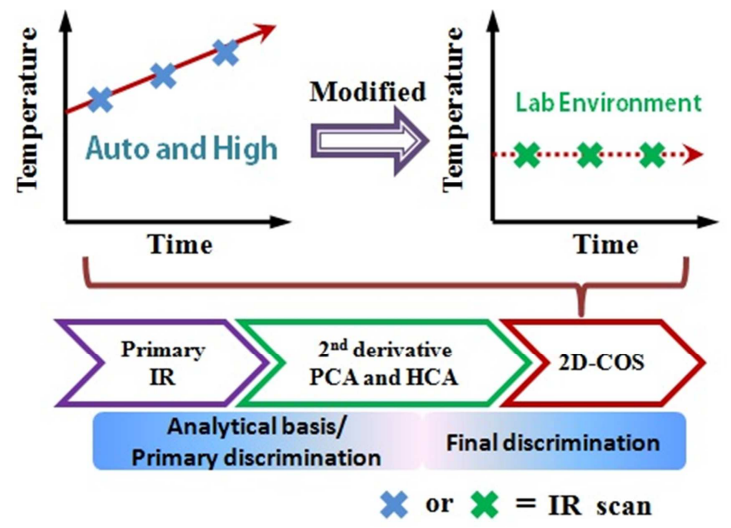

Figure 1. The principle of this work.

In this work, the lab environment (LE), in which room temperature (RT) is $25-30^{\circ} \mathrm{C}$ and humidity is $25-30 \%$, is applied as the external disturbance in the $3 \mathrm{~S}-\mathrm{IR}$ method. As shown in Figure 1, the IR data were collected at a series of intervallic time of the $\mathrm{KBr}$ pellet prepared and kept in lab environment. Different from common IR experiments in which the $\mathrm{KBr}$ pellet is tested immediately after preparation, this work used lab environment to affect the prepared $\mathrm{KBr}$ pellet to produce enough variations for the 3S-IR method. Similar to long-term stability or observation trials of drugs [21], the environmental factors were exerted onto the samples.
Using the DG-FGs of different pharmaceutical companies as the test samples, this LE was proved to be an effective external disturbance, by which the rapid discrimination of the chosen DG-FGs was achieved on the basis of the 3S-IR method.

\section{Experimental}

\subsection{Apparatus}

Spectrum 100 FT-IR spectrometer (Perkin-Elmer Inc., Waltham, Massachusetts, USA) equipped with a DTGS detector was used.

\subsection{Samples}

Samples of formula granule from 6 different pharmaceutical companies in China were collected. The pharmaceutical companies and corresponding abbreviated codes used in this article are as follows: Sichuan Neo-Green Pharmaceutical Technology Development Co., Ltd (refferred as Sample A), Guangdong Yifang Pharmaceutical Co., Ltd (refferred as Sample B), Jiangyin Tianjiang Pharmaceutical Co., Ltd (refferred as Sample C), Beijing Tcmages Pharmaceutical Co., Ltd (refferred as Sample D), Huarun Sanjiu Pharmaceutical Co., Ltd.(refferred as Sample E), China Shineway Pharmaceutical Group Ltd.(refferred as Sample F),

\subsection{Procedures and Analysis}

Each sample (about 3-5 mg) was added into $\mathrm{KBr}(200 \mathrm{mg})$. After grinded uniformly to powder, the mixture was pressed to be a $\mathrm{KBr}$ pellet for later IR test. The IR spectra of the 6 samples were accumulated by 16 times in the range of 4000 $\mathrm{cm}-1$ to $400 \mathrm{~cm}-1$ with a resolution of $4 \mathrm{~cm}-1$. The interfering signals of water and carbon dioxide were eliminated by calibration function of the IR software. The raw IR spectra were then processed by Origin software (OriginPro 2017) to substract baselines and get normalized. The resulting IR spectra (referred as primary IR spectra) were then smooth by the 4-point FFT filter method to get 2D-spectra in a rationally-selected range.

To obtain the dynamic FT-IR spectra, the prepared sample pellets were exposed in lab environment in which room temperature (RT) is $25-30^{\circ} \mathrm{C}$ and humidity is $25-30 \%$. At intervals of 30 minutes, the sample pellets were scanned with abovementioned IR parameters. After sufficient IR spectra were collected, the time-dependent spectra were processed by 2D correlation analysis software developed by Tsinghua University (Beijing, China).

\section{Results and Discussion}

\subsection{Common Features in the Primary IR Spectra}

Figure 2(a) shows the IR spectra of the 6 Danggui formula granules. Due to the same active ingredients and similar excipients, the IR spectra possess the some macroscopic characters in common. However, because of the overlapping IR spectra of the complex components, the peak cannot be 
precisely identified. In the range of 4000-2000 $\mathrm{cm}-1$, there are two identifiable peaks: the $3400 \mathrm{~cm}-1$ (the wavenumber is approximate when describing the common feature of all IR spectra, hereinafters) is attributed to $\mathrm{O}-\mathrm{H}$ stretching vibration, and the $2900 \mathrm{~cm}-1$ is attributed to $\mathrm{C}-\mathrm{H}$ antisymmetric stretching vibration, which can be attributed to the existence of excipients of polysaccharide kinds and cannot provide more information for differentiation. In the range below $800 \mathrm{~cm}-1$, the identifiable peaks are few. In the range of 1800-800 cm-1, the peaks become complex: for instance, the $1650 \mathrm{~cm}-1$ is attributed to $\mathrm{C}=\mathrm{O}$ stretching vibration, the $1450 \mathrm{~cm}-1$ is attributed to phenyl ring, the $1100-900 \mathrm{~cm}-1$ is attributed to C-O stretching vibration. The range of $1800-800 \mathrm{~cm}-1$ is of more peaks and complex peak shapes and hence can provide more details for further differentiation.

\subsection{Extended Differences in 2D Spectra and Primary Discrimination}

On the basis of the above-mentioned analysis, the range of 1800-800 cm-1 is chosen for deep differentiation. Firstly, 2D spectra are obtained as shown in Figure 2(b). Secondly, to fully reveal the differences, pattern recognition algorithm of principal component analysis (PCA) and hierarchical cluster analysis (HCA) are applied in this research. As shown in Figure 3(a), the first two PCA factors total to more than $90 \%$, and hence the PCA is qualified to express the main features for discrimination. Sample D is clearly different from all the others. Sample A and B are of delicate difference and are close in PCA figure. Sample C, sample D, sample E and the Sample $\mathrm{A} / \mathrm{B}$ group are discriminated by a moderate distance in PCA figure from one another. To further analyze the inter-similarity, HCA is applied as shown in Figure 3(b), sample C and E are firstly clustered and then clustered with sample A. Then sample B and F are cluster to this group in order. Sample D is the last one. PCA and HCA convert the 2D spectra to visualized differences and inter-similarity, which not only functions to contribute to the primary discrimination of the 6 samples, but also prove the 2D spectra of the chosen range include sufficient information for discrimination and thus provide the basis for 2D-COS IR method.

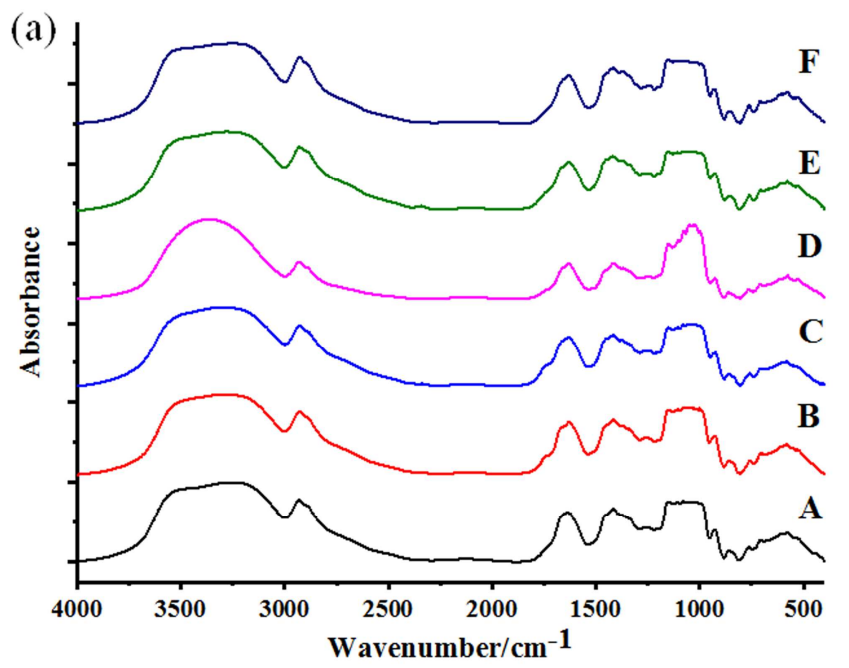

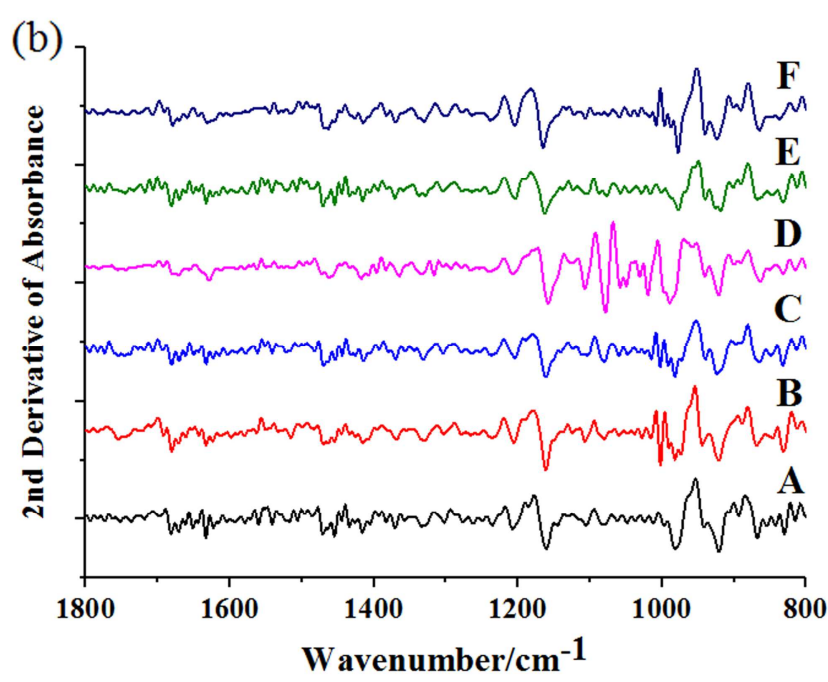

Figure 2. (a) IR and (b) $2 D$ spectra (1800-800 $\left.\mathrm{cm}^{-1}\right)$ of the 6 Danggui formula granules. A-E respectively represents Sample A-E.

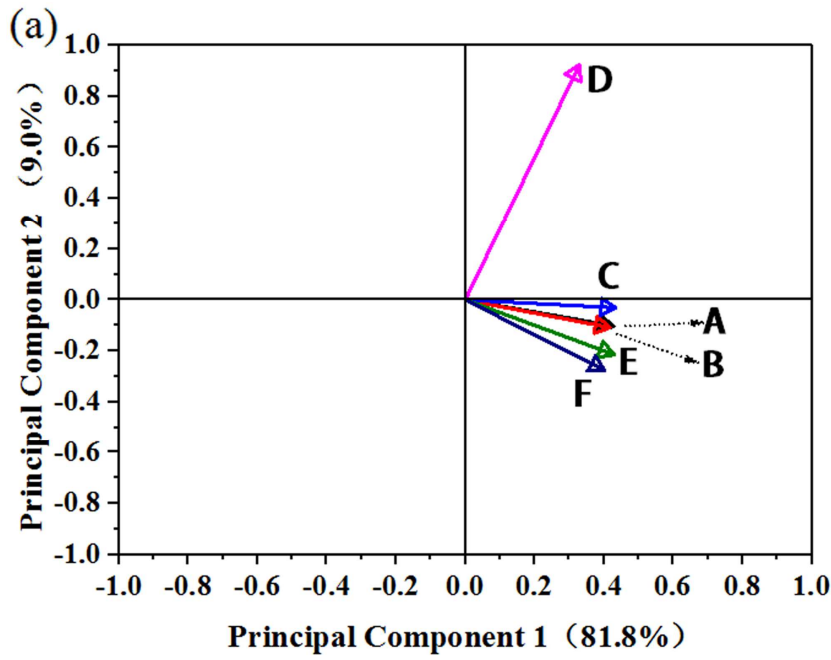

(b)

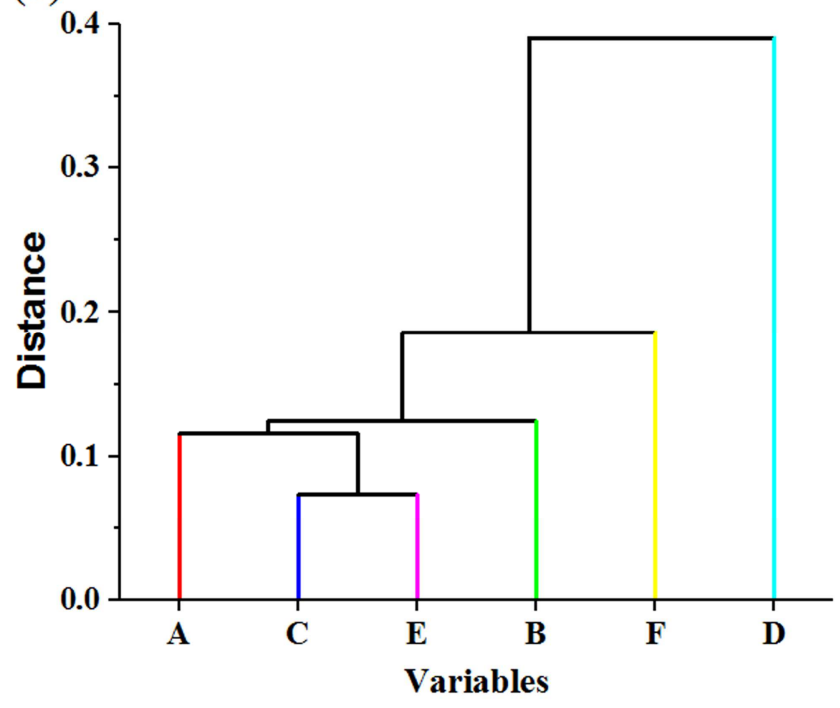

Figure 3. (a) PCA and (b) HCA of $2 D-I R$. 


\subsection{LE-Based 2D-COS IR for Final Discrimination}

The LE-based synchronous 2D-COS IR are obtained from the range of $1800-800 \mathrm{~cm}-1$. In the $2 \mathrm{D}-\mathrm{COS} \mathrm{IR}$, positive correlation (red or green area) means that a group of absorption bands changes simultaneously (either increase or decrease); negative correlation (blue area) is just the reverse [19]. As shown in Figure 4, the macroscopic and discriminative features are very clear. The strongest positively-correlated peak group (red area) is around 1000 $\mathrm{cm}-1$, which can be seen in all samples. The negatively-correlated peaks (blue area) contribute differently, which can help discriminate all the samples. Sample B and F are obviously different from the others. Sample A and C, sample D and E are two close pairs and meanwhile possess subtle different patterns of blue area between each other. Therefore, the LE is proved to an effective external disturbance in 3S-IR method. As a result, the 6 samples of Danggui Formula Granules can be completely discriminated.

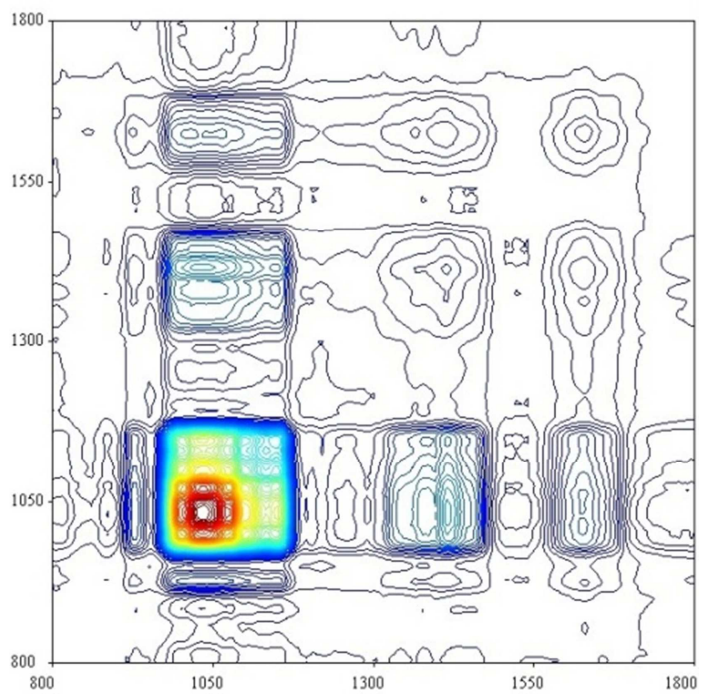

(a)

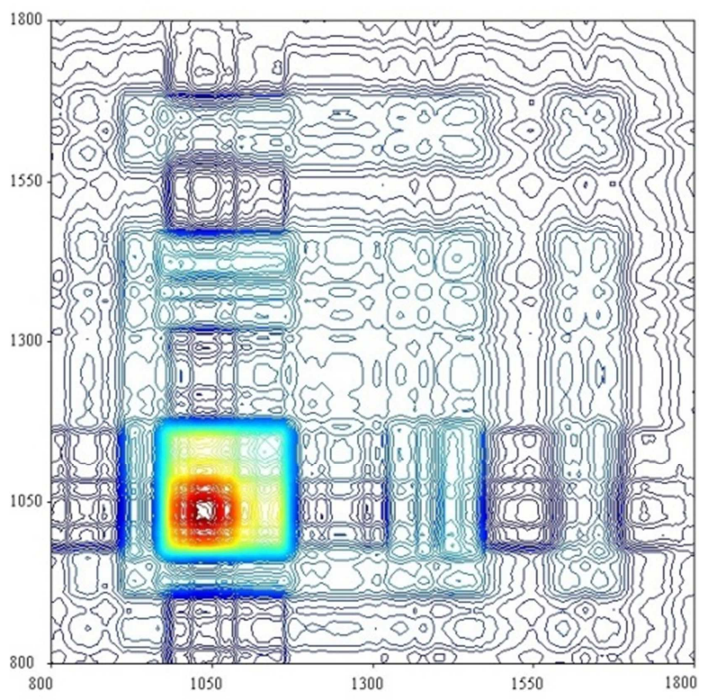

(b)

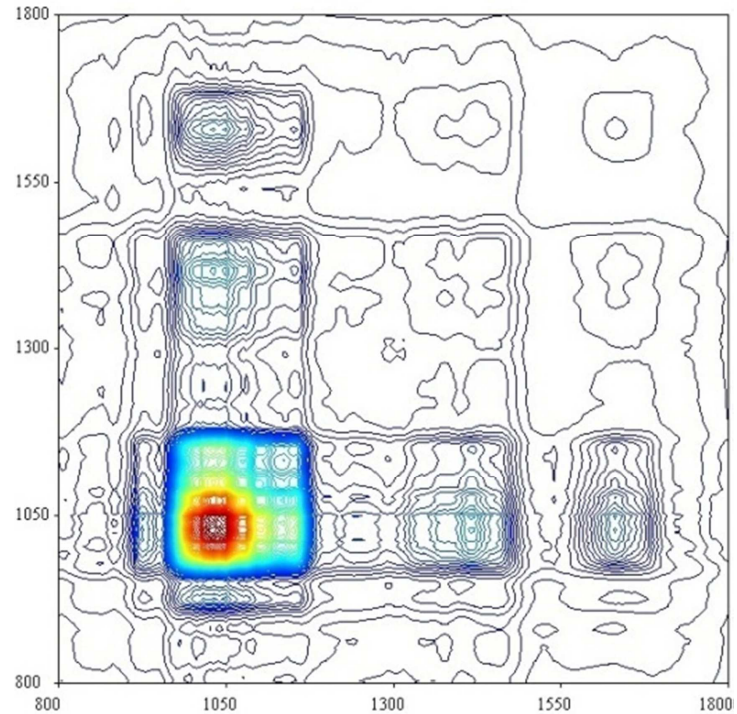

(c)

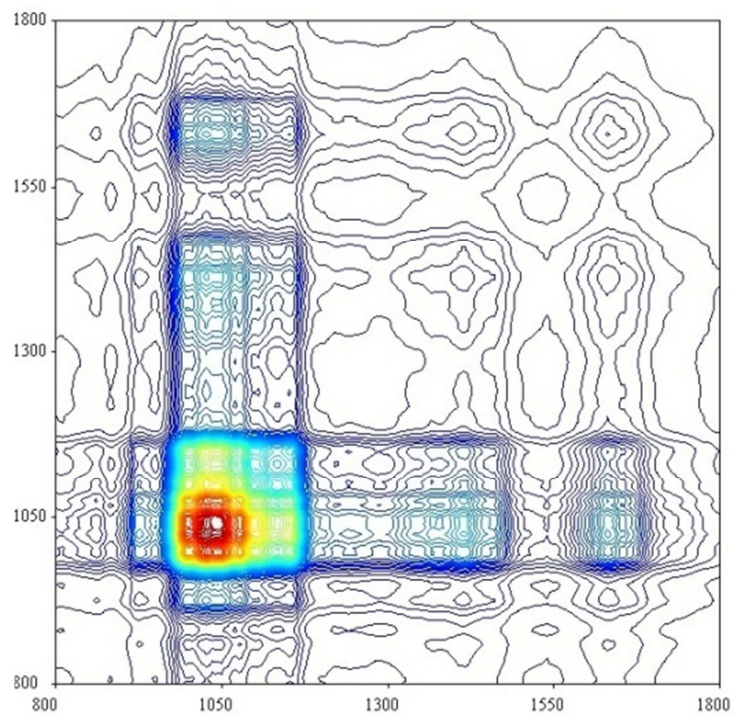

(d)

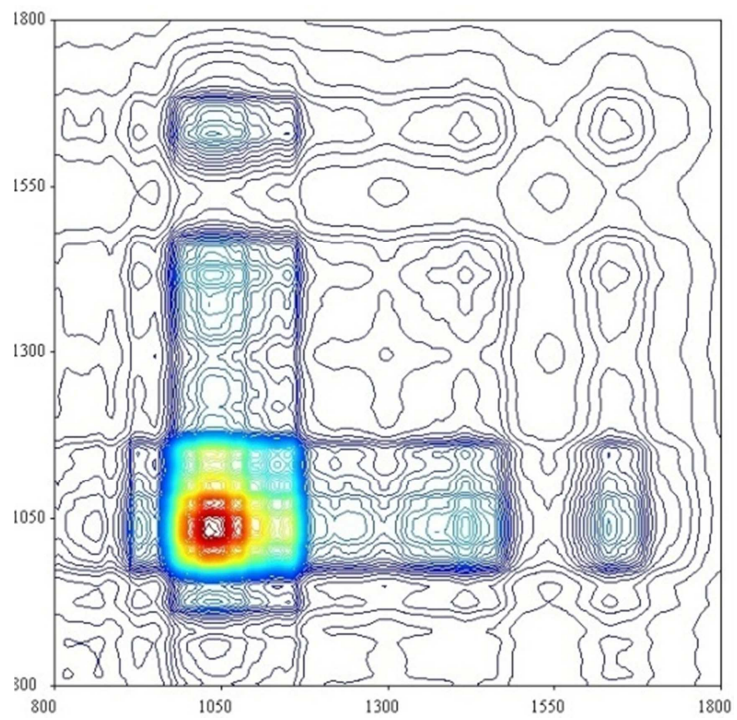

(e) 


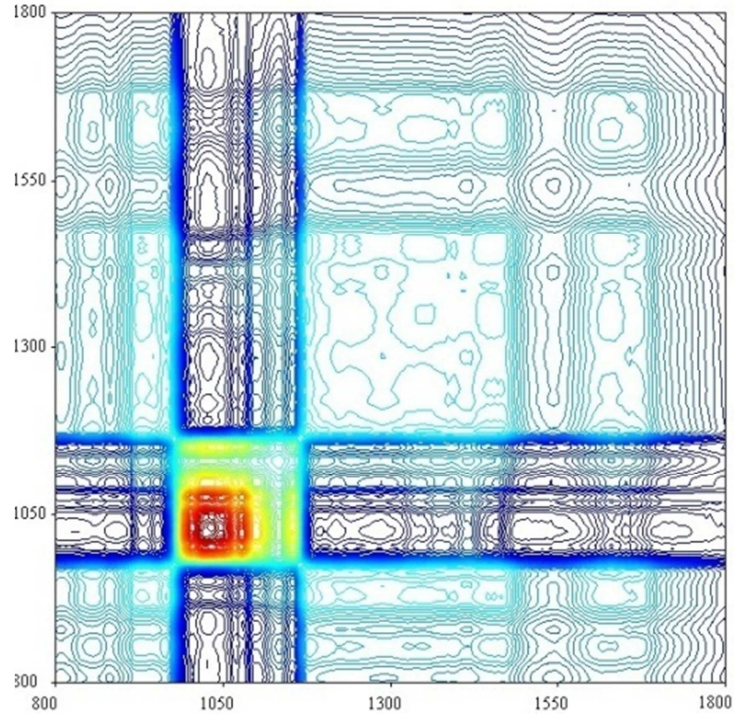

(f)

Figure 4. LE-based 2D-COS IR. (a) (f) represent Sample A-F.

\section{Conclusion}

In this work, LE is applied as the external disturbance in the 3S-IR method. Firstly, the 2D-spectra and pattern recognition algorithm of PCA and HCA are used to select the analytical basis for 2D-COS IR and accomplish the primary discrimination. Secondly, LE-based 2D-COS IR achieves the final discrimination of different Danggui Formula Granules. LE is proved to be an effective external disturbance and avoid the complex parts of programmed temperature and sampling in IR instrument. As a consequence, the LE improved 3S-IR method can be applied in TCM analysis.

\section{Acknowledgements}

This work is sponsored by the Key Scientific Project of University in Hebei Province (No. ZD2015001). PhD fund of Hebei University of Chinese Medicine (No. BSZ2017002) and National Science Youth Fund (No. 21705032).

\section{References}

[1] The State Pharmacopoeia Commission of People's Republic of China, Pharmacopoeia of the People's Republic of China, vol. 1, Chemical Industry Press, Beijing, China, 2015, p. 133.

[2] B. X. Li, C. Q. Wang, L. L. Xi, Y. H. Wei, H. G. Duan and X. N. Wu. Anal. Methods, 2014, 6, 9691-9697.
[3] X. P. Chen, W. Li, X. F. Xiao, L. L. Zhang and C. X. Liu, Chinese Journal of Natural Medicines, 2013, 11, 577-587.

[4] Y. C. Wu and C. L. Hsieh, Chinese Medicine, 2011, 6, 32-37.

[5] X. Fu, Q. H. Wang, Z. B. Wang, H. X. Kuang and P. H. Jiang, Aging And Disease, 2016, 7, 502-513.

[6] Y. Jin, C. Qu, Y. P. Tang, H. Q. Pang, L. L. Liu, Z. H. Zhu, E. X Shang, S. L. Huang, D. Z. Sun and J. A. Duan, J. Ethnopharmacol., 2016, 181, 158-171.

[7] H. W. Lee, J. H. Jun, K. J. Kil, B. S. Ko, C. H. Lee and M. S. Lee, Maturitas, 2016, 85, 19-26.

[8] J. B. Chen, S. Q. Sun and Q. Zhou, Anal. Methods, 2016, 8, 8329-8336.

[9] L. Y. Niu, Research on Quality of Traditional Chinese Medicine Formula Granule, Science Press, Beijing, China, 2017.

[10] Technical Requirements of Quality Control and Standard Establishment for Traditional Chinese Medicine Formula Granule (Exposure Draft), National Pharmacopoeia Commission, China, 2016.

[11] Y. Jiang, B. David, P. F. Tu and Y. Barbin, Anal. Chim. Acta, 2010, 657, 9-18.

[12] W. Tian, Y. Q. Zhen, S. S. Fan, Y. H. Dou, Z. Chen and L. Y. Niu, Chinese Journal of Pharmaceuticals, 2018, 53, 140-144.

[13] Y. R. Tian, J. M. Ma, X. G. Wang, J. S. Li and L. Y. Niu, Chinese Traditional Patent Medicine, 2017, 39, 1845-1849.

[14] Y. Q. Zhen, W. Tian, Y. C. Wang, G. Z. Jiang and L. Y. Niu, Chinese Journal of Experimental Traditional Medical Formulae, 2017, 23, 79-83.

[15] S. Q. Sun, J. B. Chen, Q. Zhou, G. H. Lu and K. Chan, Planta Medica, 2010, 76, 1987-1996.

[16] A. X. Liu, J. J. Wang, Y. Z. Guo, Y. Xiao, Y. Wang, S. Q. Sun and J. B. Chen, Spectrochim. Acta. A, 2018, 192, 93-100.

[17] L. Xiang, J. J. Wang, G. J. Zhang, L. X. Rong, H. Z. Wu, S. Q. Sun, Y. Z. Guo, Y. F. Yang, L. N. Lu and L. Qu, J. Mol. Struct., 2016, 1124, 164-172.

[18] J. B. Chen, B. L. Guo, R. Yan, S. Q. Sun and Q. Zhou, Spectrochim. Acta. A, 2017, 182, 81-86.

[19] Y. Z. Guo, B. R. Lv, J. J. Wang, Y. Liu, S. Q. Sun, Y. Xiao, L. N. Lu, L. Xiang, Y. F. Yang, L. Qu and Q. H. Meng, Spectrochim. Acta. A, 2016, 153, 550-559.

[20] C. H. Xu, Y. Wang, J. B. Chen, Q. Zhou, P. Wang, Y. Yang and S. Q. Sun, J. Pharmaceut. Biomed, 2013, 74, 298-307.

[21] ICH, Q1E, Evaluation for stability data, February, 2003. 